\title{
Nanoparticles of amorphous cellulose and their properties
}

\author{
Michael Ioelovich \\ Dept. of Chemistry, Designer Energy Ltd, Rehovot, Israel
}

Email address:

Bd895892@zahav.net.il(M. Ioelovich)

\section{To cite this article:}

Michael Ioelovich. Nanoparticles of Amorphous Cellulose and Their Properties. American Journal of Nanoscience and Nanotechnology. Vol. 1, No. 1, 2013, pp. 41-45. doi: 10.11648/j.nano.20130101.18

\begin{abstract}
Method of preparation and some properties of amorphous cellulose nanoparticles (ANP) have been described in this paper. It was shown that ANP have spherical shape and are characterized by high degree of pantamorphia, low DP and increased content of sulfonic groups. The amorphous nanoparticles of cellulose are completely hydrolyzed by cellulolytic enzymes with forming of glucose. Concentrated paste of ANP has expressed thickening properties and therefore its additive can prevent phase separation of water dispersions of various substances. Low-acidic and soft nanoparticles can be used in cosmetic formulation for gentle skin peeling. Moreover, due to increased content of acidic functional groups, ANP can immobilize various therapeutically-active substances (TAS) containing basic functional groups. The ANP-TAS complexes can be used in remedies aimed for effective care and cure of the skin.
\end{abstract}

Keywords: Amorphous Cellulose, Nanoparticles, Isolation, Properties, Applications

\section{Introduction}

As known, ultra small nanomaterials have unique physico-chemical properties, such as developed specific surface, high bonding ability and increased reactivity, which are different from macroscopic materials of the same composition $[1,2]$. These properties significantly expand application areas of the nanomaterials. Currently the main technical applications of nanomaterials are in the fields of nanocomposites, nanochips, nanosensors, nanoscale labels and indicators. In cosmetics nanoparticles are applied mainly in creams, e.g. for sun protection. In pharmaceutics and medicine nanomaterials can be used as carriers for drug delivery, implantants, as well as for advanced treatment and diagnostic methods, etc.

The visible advantages of the nanomaterials in technical branches can turn to drawbacks, when use these materials for cure and care of humans [3-5]. Small nanoparticles penetrate trough cell membrane, accumulate and damage functions of organelles, cells and organs. The high reactivity and catalytic ability of inorganic nanomaterials causes generation of active oxygen and free radicals that injure proteins, RNA and DNA. Decomposition of phagocytes at their interaction with alien nanoparticles leads to forming of toxic products that poison the living organisms. Indeed, it was discovered that most of inorganic nanomaterials (e.g. nanoparticles of silver, gold, cooper, titan dioxide, silica dioxide, zinc oxide, carbon nanotubes, etc) even at small doses are harmful and dangerous for organisms [6-10]. Negative effect of nanoparticles rises with decreasing of particle size [11]. Many organic nanomaterials made of synthetic polymers also can be danger. Even biological nanoparticles such as well-known liposomes that are investigated during 20 years, not have been approved for use in clinic practice [12].

Among various organic materials, namely cellulose is the most appropriate for preparation of various types of nanomaterials, since this nanostructured natural polymer has unique properties such as low density, hardness and abrasivity; ability to structural and chemical modification; biocompatibility; biodegradability in the nature, etc $[2,13$, 14].

For a long time, cellulose materials are used in medicine and biology. Analogue of nanocellulose - microcrystalline cellulose, is ingredient of tablets and other drug forms [14]. Cellulose is the most widespread and renewable natural organic polymer. Resource of cellulose in the nature increases by means of biosynthesis on 75 billion tons yearly. Therefore, production of cellulose nanomaterials has unlimited source and can be inexpensive.

Currently, two main types of nanocellulose are known: crystalline nanowhiskers and nanofibers [13-19]. Main application field of these nanomaterials is production of nanopaper and reinforced polymer nanocomposites. It should be noted that nanowhiskers have shape of acicular 
nanocrystals that can injure the cells and tissues; these nanoparticles probably are not suitable for medicine and cosmetics. An additional problem that organism of people doesn't contain cellulolytic enzymes. Therefore, it is dangerous to introduce the cellulose nanomaterials into digestive or blood systems or into other organ of human. In the most cases only external application of the nanocellulose is possible. The method of isolation and main properties of special spherical nanoparticles made of amorphous cellulose were described in this paper. Potential application areas of these nanoparticles were discussed.

\section{Experimental}

\subsection{Material}

Initial cellulose material was MCC Avicel PH-301 made of FMC BioPolymer Co. This MCC type has average particles size of $50 \mu \mathrm{m}$ and $\mathrm{DP}=170$. Chemical pure $95 \mathrm{wt} . \%$ sulfuric acid was supplied from Sigma-Aldrich Co. The concentrated acid was diluted drop-wise with water at cooling in an ice bath up to $80 \mathrm{wt} . \%$ (density $1.727 \mathrm{~g} / \mathrm{cm} 3$ ).

Biological and therapeutically-active substances were supplied also from Sigma-Aldrich Co, and namely: Gelatin (Bloom strength 300) and Trypsin from bovine pancreas (6000 BAEE units/mg).

\subsection{Acidic Treatment}

Initial MCC was mixed with water in a lab glass, then 80 wt.\% sulfuric acid (SA) was slowly added at cooling to obtain the required final concentration (50 to $70 \mathrm{wt} . \%$ ) and acid/cellulose ratio 10 . The glass was placed into water bath at room temperature $\left(25^{\circ} \mathrm{C}\right)$ and stirred for $1 \mathrm{~h}$. After acidic treatment the contents of the glass were poured out into tenfold volume of cold water at stirring to regenerate the dissolved amorphous cellulose. Cellulose was separated from liquid by centrifugation at $\mathrm{g}=4000$ for $10-15 \mathrm{~min}$; washed with distillated water to $\mathrm{pH} \mathrm{5,} \mathrm{separating} \mathrm{from}$ water by centrifugation.

Yield of amorphous cellulose (YA) was calculated as follows:

$$
\mathrm{YA}, \%=\mathrm{R}\left(\frac{W}{W_{o}}\right)
$$

where $W_{o}$ is dry weight of the initial MCC-sample; $W$ is weight of solids in the sediment; and $R$ is percentage of amorphous cellulose regenerated from acidic solutions.

\subsection{Sorption of Therapeutically-Active Substance (TAS)}

Cellulose sediment was introduced into $1 \%$ solutions of TAS - proteolytic enzyme Trypsin, at liquid/solid ratio 20. The mixture was kept overnight at stirring at room temperature and then centrifuged to isolate the cellulose sediment containing the sorbed TAS. Content of the TAS sorbed by cellulose was determined as:

$$
\mathrm{S}, \mathrm{mg} / \mathrm{g}=\mathrm{V}\left(\mathrm{C}_{\mathrm{o}}-\mathrm{C}\right) / \mathrm{W}_{\mathrm{s}}
$$

where Ws is solid content of the sediment; V is volume of TAS-solution; $\mathrm{Co}$ is initial concentration of the solution, and $\mathrm{C}$ is concentration of the solution after sorption measured by refractometry method.

\subsection{Preparation Dispersions of Cellulose Particles}

Cellulose sediment before and after sorption of TAS was diluted with distillated water to solid concentration about $1 \%$ and disintegrated by ultrasound disperser "Branson S450CE" at $20 \mathrm{kHz}$ for $10-15 \mathrm{~min}$.

To increase concentration of cellulose particles the diluted dispersion was evaporated in vacuum at $50^{\circ} \mathrm{C}$ to final concentration about $10-15 \%$.

\subsection{Preparation Dry Cellulose Particles}

To obtain dry particulate cellulose the concentrated water dispersion having about $10-15 \%$ solids was washed with absolute ethanol, acetone and hexane, separating from solvent at the each washing by centrifugation, and finally dried at $50^{\circ} \mathrm{C}$ to constant weight.

\subsection{Enzymatic Hydrolysis}

Cellulose samples were hydrolyzed with cellulolytic enzymes according to protocol described in paper [20].

\subsection{X-Ray Diffraction}

A Rigaku-Ultima Plus diffractometer $(\mathrm{CuK} \alpha$ - radiation, $\lambda=0.15418 \mathrm{~nm})$ was used for X-ray investigations. Diffractograms of dry cellulose particles were recorded in the $\varphi=2 \Theta$ angle range from 5 to $80^{\circ}$. After recording of the diffractograms, the background was separated, and selected X-ray patterns were corrected and normalized. Then diffraction intensities from crystalline and non-crystalline regions were separated by a computerized method. The degree of crystallinity (X) and pantamorphia (A) were calculated according to following equations $[21,22]$ :

$$
\begin{aligned}
& X, \%=100 \frac{\int I_{C} d \varphi}{\int I_{o} d \varphi} \\
& A, \%=100-X
\end{aligned}
$$

where Ic and Io are the corrected and normalized intensities of diffraction from the crystalline part and whole sample, respectively

\subsection{Average Degree of Polymerization}

The average degree of polymerization (DP) was measured by the viscosity method using diluted solutions of dry cellulose particles in Cadoxen [20].

\subsection{Determination of Functional Groups}

Content of sulfonic groups in acid-treated MCC was calculated from results of sulfur assay that was performed by means of elemental analyzer FLASH-2000. Content of reducing groups in the samples was found from results of 
determination of the cooper number by standard TAPPI method T430.

\subsection{SEM}

Shape and size of particles were investigated by SEM "Hitachi S-4700." The diluted dispersion of cellulose particles was subjected to ultrasonic treatment for $5 \mathrm{~min}$. A drop of the diluted dispersion was applied onto a substrate and dried and the dry sample was coated with a thin layer of gold. Then the sample was placed in the microscope, evacuated, and electronic image of particles was obtained.

\subsection{Rheological Properties}

Viscosity of water dispersions of cellulose particles were studied by Brookfield DV-II+ viscometer at $25^{\circ} \mathrm{C}$.

\section{Results and Discussion}

XRD-investigations of isolated cellulose particles showed that the amorphous phase state of the particles can be achieved, when concentration of SA was higher than 65 wt.\% (Fig. 1).

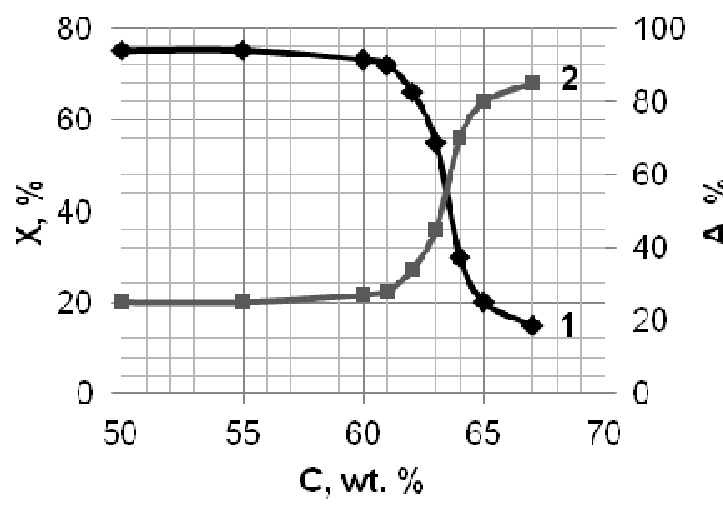

Figure 1. Dependence of crystallinity (1) and degree of pantamorphia (2) of isolated cellulose particles on concentration of sulfuric acid

In contrast to initial crystalline MCC sample, the cellulose particles prepared by regeneration from 65-67 wt.\% SA possessed of diffuse X-ray diffractogram typical for the amorphized polymers (Fig. 2).

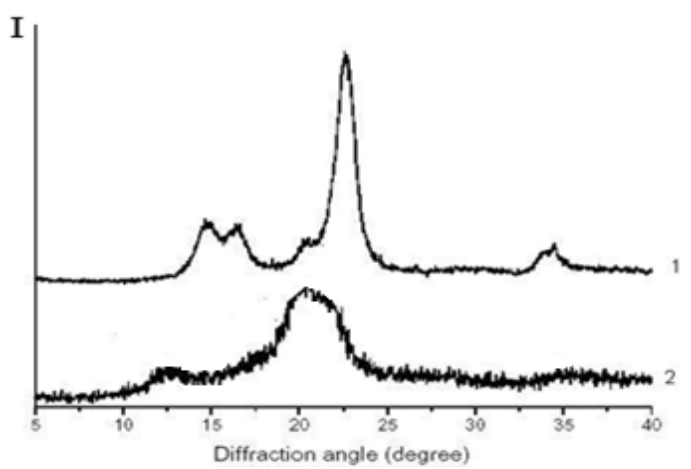

Figure 2. X-ray diffractograms of crystalline MCC (1) and amorphous cellulose particles (2)
Maximal yield of amorphous cellulose about $65 \%$ was achieved after regeneration from solutions in 65-66 wt.\% SA (Fig 3). Increasing of SA concentration more than 66 wt. \% leaded to decrease in yield of the regenerated amorphous cellulose, and after treatment of the initial MCC sample with 70 wt. \% SA the dissolved cellulose cannot be regenerated from the acidic solution by diluting with water due to fast acidic depolymerization of cellulose and forming the water-soluble oligomers [23].

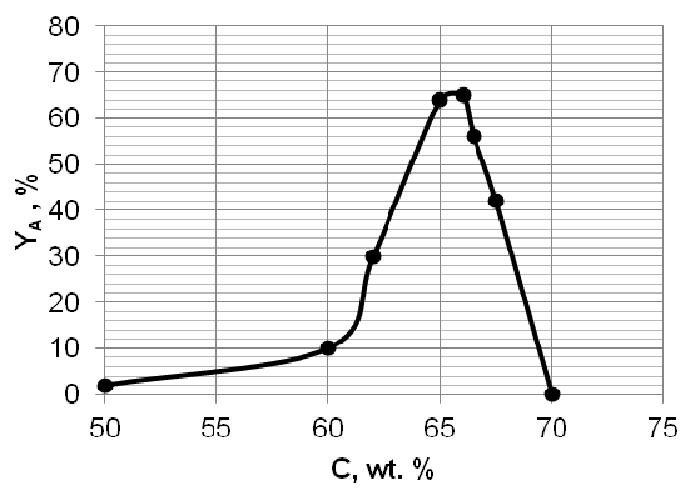

Figure 3. Yield of amorphous cellulose as function of SA-concentration

The initial crystalline cellulose sample - MCC has coarse particles with average size about of $50 \mu \mathrm{m}$ (Fig. 4a). The amorphous cellulose after high-power disintegration of in the water medium turns to spherical nanoparticles with diameter of 100-200 nm (Fig. 4 b).
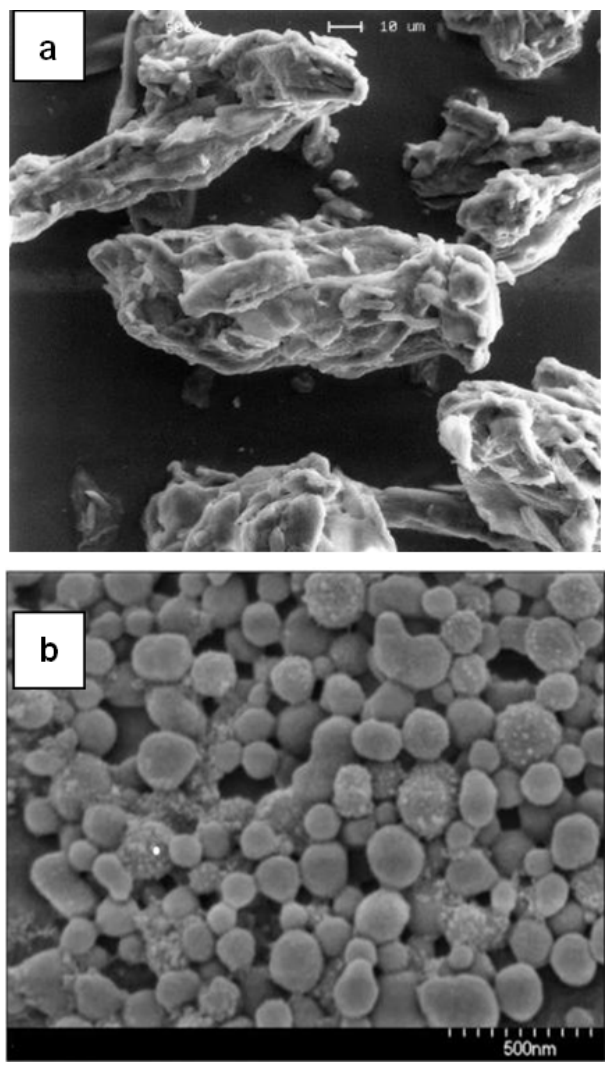

Figure 4. SEM image of the initial MCC sample (a) and nanoparticles of the amorphous cellulose (b) 
The isolated amorphous nanoparticles (ANP) are characterized by high degree of pantamorphia, increased yield and decreased degree of polymerization (Table 1). Moreover, these particles contain sulfonic and reducing groups.

Table 1. Main features of isolated cellulose nanoparticles

\begin{tabular}{ll}
\hline Feature & Value \\
\hline Phase state & Amorphous \\
Degree of pantamorphia & $80-85 \%$ \\
Average diameter of particles & $100-200 \mathrm{~nm}$ \\
Average DP & $70-80$ \\
Content of sulfonic groups & $200-230 \mathrm{meq} / \mathrm{kg}$ \\
Content of reducing groups & $82-88 \mathrm{meq} / \mathrm{kg}$ \\
Yield & $64-66 \%$ \\
\hline
\end{tabular}

Due to amorphous nature these nanoparticles are completely hydrolyzed by cellulolytic enzymes and turn into glucose with about $100 \%$ yield (Fig. 5).

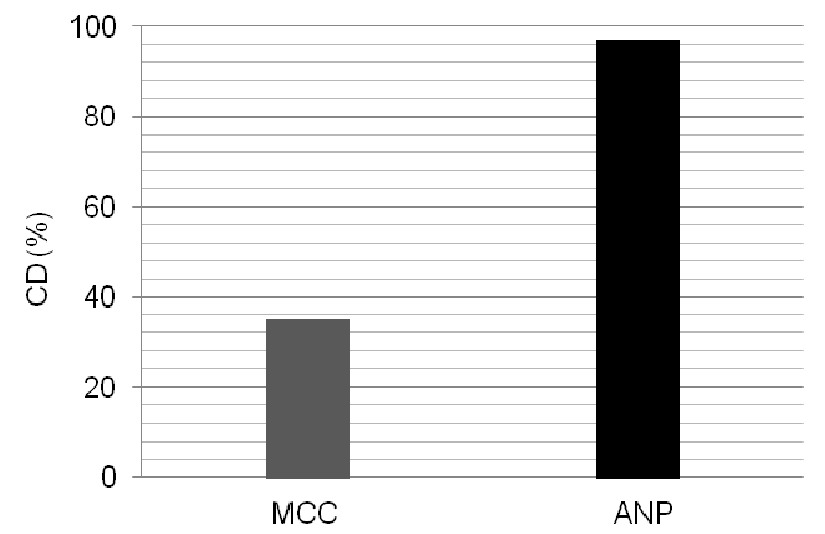

Figure 5. Conversion degree (CD) of the initial MCC sample and amorphized cellulose nanoparticles (ANP) after enzymatic hydrolysis of the samples during $24 \mathrm{~h}$.

Study of water dispersions contained ANP showed that with increasing of ANP-concentration viscosity of the dispersion rises (Fig. 6). When the concentration of ANP achieves $10 \%$, the dispersion turns to gel-like thick paste.

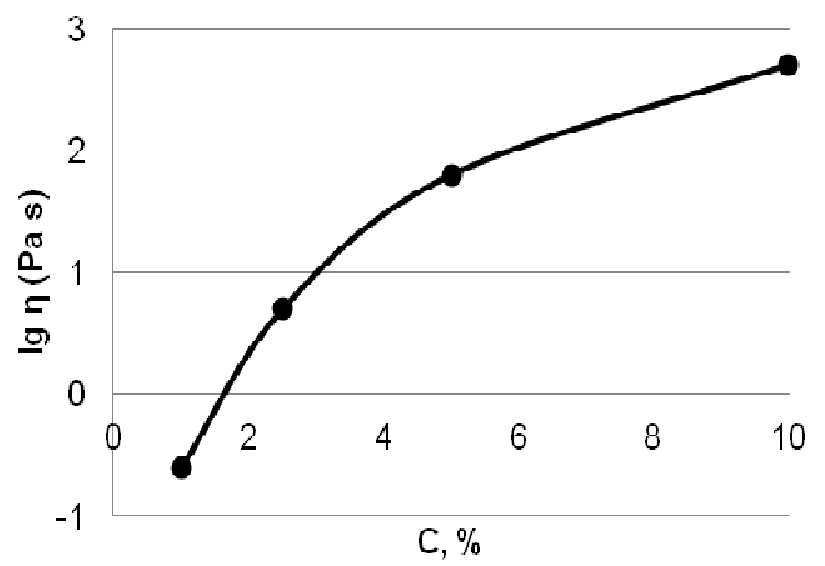

Figure 6. Dependence of viscosity of water dispersion on concentration of ANP at shear rate $1, s^{-1}$
Due to expressed thickening properties, low additive of ANP can prevent phase separation of water dispersions of some TAS (Fig. 7).

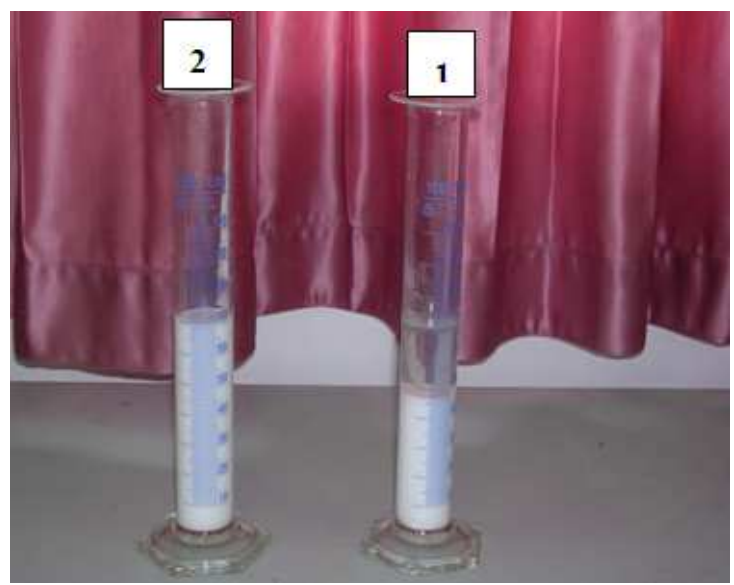

Figure 7. Separated original drug "Maalox" (1) and stable modified "Maalox”, containing 2\% of ANP (2)

After sorption experiments it was established that about $0.25 \mathrm{mg}$ of Trypsin were immobilized by $1 \mathrm{~g}$ of amorphous cellulose. The product was dispersed in water to obtain $1 \%$ dispersion of nanoparticles and then evaporated to about $10 \%$ solid content. The gelatin-gel assay was used to check activity of the immobilized Trypsin. About $5 \mathrm{~g}$ of $10 \%$ ANP-Trypsin paste was mixed with $5 \mathrm{~g}$ of $5 \%$ gelatin solution in $1 \%$ sodium bicarbonate at $40^{\circ} \mathrm{C}$; then the mixture was cooled in refrigerator to gelation. The chemical glass containing the solid gel was put to water bath and kept at $25^{\circ} \mathrm{C}$.

After about $2 \mathrm{~h}$ the gel was decomposed and turned into liquid sol under effect of the immobilized proteolytic enzyme (Fig. 8).

This test evidences about keeping the enough high activity of the TAS after its immobilization by ANP.

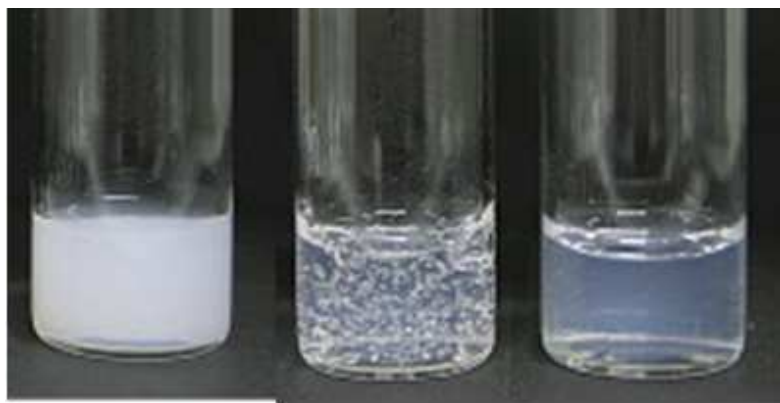

(a)

(b)

(c)

Figure 8. Liquefaction of solid gelatin gel under effect of ANP-Trypsin complex: (a) initial solid gel containing ANP-Tr; (b) starting of the gel liquefaction; (c) completely liquefaction of the gel

\section{Conclusion}

Method of preparation and some properties of the new nanocellulose type - amorphous cellulose nanoparticles 
(ANP), were described. It was shown that ANP have spherical shape and are characterized by high degree of pantamorphia and increased content of sulfonic groups. The amorphous nanoparticles are completely hydrolyzed by cellulolytic enzymes with forming of glucose that can be used further for biological, cosmetic or medical application. Concentrated paste of ANP has expressed thickening properties and therefore its additive can prevent the phase separation of various water dispersions. Low-acidic and soft nanoparticles can be used in cosmetic formulation for gentle skin peeling. Moreover, due to increased content of acidic functional groups, ANP can immobilize proteolytic enzymes and other therapeutically active substances (TAS) containing basic functional groups. The ANP-TAS complexes can be applied in remedies aimed for effective care and cure of the skin. In particular, ANP-Trypsin complex can remove dead skin cells and clean the wounds without damage of healthy tissues.

\section{References}

[1] Ch. Hayashi, R. Uyeda, and A. Tasaki, Ultra-fine Particles: Exploratory Science and Technology, Westwood: Noyes Publications, 1997.

[2] M. Ioelovich, "Cellulose as a nanostructured polymer," Bioresources, vol. 3, pp. 1403-1418, 2008.

[3] V.E. Kagan, H. Bayir, and A.A. Shvedova, "Nanomedicine and nanotoxicology: two sides of the same coin," Nanomedicine: nanotechnology, biology and medicine, vol.1 p.313-316, 2005.

[4] C. Buzea, I. Pacheco and K. Robbie, "Nanomaterials and nanoparticles: sources and toxicity," Biointerphases, vol. 2, p.17-71, 2007.

[5] K. Wise and M. Brasuel, "The current state of engineered nanomaterials in consumer goods and waste streams," Nanotech. Sci. Appl., vol.4, pp.73-86, 2011.

[6] V. Alt, T. Bechert, P. Steinrücke, M. Wagener, P. Seidel, E. Dingeldein , E. Domann and R. Schnettler, "An in vitro assessment of the antibacterial properties and cytotoxicity of nanoparticulate silver bone cement," Biomaterials, vol. 25, pp. 4383-4391, 2004.

[7] N. Lewinski, V. Colvin and R. Drezek, "Cytotoxicity of nanoparticles,” Small Journal, vol. 4, pp. 26- 49, 2008.

[8] Z. Chen, H. Meng, G. Xing, C. Chen, Y. Zhao, G. Jia, T. Wang, H. Yuan, C. Ye, F. Zhao and Z. Chai, "Acute toxicological affects of copper nanoparticles in vivo," Journal of Physic. Chem, Toxicol. Letters, vol. 163, pp.109-120, 2006.

[9] N. Lu, "Nano titanium dioxide photocatalytic protein tyrosine nitration: a potential hazard of $\mathrm{TiO} 2$ on skin," Biochem. Biophys. Res. Commun., vol. 370, pp.675-680, 2008.

[10] K. Donaldson, R. Aitken, L. Tran, V. Stone, R. Duffin, G. Forrest and A. Alexander, "Carbon nanotubes: review of their properties in relation to pulmonary toxicology and workplace safety," Toxicological Science, vol. 92, pp. 5-22, 2006.

[11] J. Jiang, G. Oberdrster, A. Elder, R. Gelein, P. Mercer and P. Biswas, "Does nanoparticle activity depend upon size and crystal phase?" Nanotoxicology, vol. 2, p. 33- 42, 2008.

[12] L. Zhang, F.X. Gu, J.M. Chan, A.Z. Wang, R.S. Lange and O.C. Farokhzad, "Nanoparticles in medicine: therapeutic applications and developments," Clinical Pharma. Therap., vol. 83, pp.761-769, 2008.

[13] M. Ioelovich, O. Figovsky and A. Leykin, "Nanocellulose and its applications," Proceedings of 7th World Congress "Nanocomposite-2007", Las Vegas, USA, 2007.

[14] M. Ioelovich and A. Leykin, "Microcrystalline cellulose: nanostructure formation," Cellulose Chem. Technol., vol.40, pp. 313-317, 2006.

[15] D. Klemm, D. Schumann, F. Kramer, N. Hessler, M. Hornung, H-P. Schmauder and S.Marsch, "Nanocelluloses as innovative polymers in research and application," Adv. Polymer Sci., vol. 205, pp.49-96, 2006.

[16] M. A. Hubbe, O.J. Rojas, L.A. Lucia and M. Sain, "Cellulosic nanocomposites - a review," Bioresources 3(3), pp. 929-980, 2008.

[17] Y. Habibi, L. A. Lucia and O. J. Rojas, "Cellulose nanocrystals: chemistry, self-assembly, and applications," Chemical Reviews, vol. 110, pp. 3479-3500, 2010.

[18] Y. Li and A. Ragauskas, "Cellulose nano-whiskers as a reinforcing filler in polyurethanes," Advances in Diverse Industrial Applications of Nanocomposites, vol. 3, pp. 17-36, 2011.

[19] I. Siro and D. Plakett, "Microfibrillated cellulose and new composite materials," Cellulose, vol. 17, pp.459-494, 2010.

[20] M. Ioelovich, "Study of cellulose interaction with concentrated solutions of sulfuric acid," ISRN Chem. Eng., vol. 2012, pp. 1-7, 2012.

[21] M. Ioelovich, "Optimal conditions for isolation of nanocrystalline cellulose particles," Nanosci. Nanotech., vol.2, pp.9-13, 2012.

[22] M. Ioelovich, A. Leykin and O. Figovsky, "Study of cellulose paracrystallinity,” Bioresources, vol. 5, pp. 1393-1407, 2010.

[23] F. Camacho, P. Gonzalez-Tello, E. Jurado and A. Robles, "Microcrystalline cellulose hydrolysis with concentrated sulphuric acid," Journal Chem. Technol. Biotechnol., vol. 67, pp. 350-356, 1996. 